\title{
UN ENFOQUE ACTUAL SOBRE LA FIBROTOMÍA COMO TERAPIA PARA EVITAR RECIDIVA EN LOS TRATAMIENTOS DE ORTODONCIA. UNA REVISIÓN
}

\author{
FIBROTOMY AS A THERAPY TO AVOID RECURRENCE IN ORTHODONTIC \\ TREATMENTS. A REVIEW \\ Sven Mario Anaya Espinoza ${ }^{1}$ \\ Sven_anaya@usmp.pe \\ ORCID: 0000-0002-4353-151X
}

\section{RESUMEN}

Esta investigación se basa en la revisión de un tratamiento coadyuvante a la contención fija o removible postratamiento de ortodoncia para reducir la recidiva. La recidiva postratamiento de ortodoncia es un problema frecuente sobre todo en pacientes que tuvieron las piezas dentarias muy giradas o inclinadas, incluso en pacientes portadores de aparatos de contención fija o removible. Actualmente, se consideran a las fibras transeptales como causantes de las recidivas, especialmente las oxitalánicas por la posición que adoptan desde la unión cemento-adamantina de un diente hasta la misma unión del diente contiguo pasando sobre la cresta alveolar y por sus características elásticas. La fibrotomía circunferencial supracrestal (FCS) surge como una terapia coadyuvante para reducir las tasas de recidivas postratamiento de ortodoncia. El objetivo de la presente revisión de la literatura es describir el enfoque actual sobre la fibrotomía como terapia eficiente y estable a largo plazo para evitar la recidiva en los tratamientos de ortodoncia. La técnica quirúrgica no ha sufrido variaciones, pero sí han surgido autores que buscan realizar la FCS con diferentes instrumentos, tales como la electrocirugía y el láser de diodos de baja intensidad. A largo plazo, no existen diferencias significativas entre los diferentes instrumentos utilizados para la FCS comparados con la convencional, pero sí existen diferencias significativas entre realizar la FCS y no realizarla. La FCS es una terapia eficiente y estable sin importar el instrumento utilizado.

Palabras clave: ortodoncia, fibrotomía circunferencial superficial, recidiva, fiberotomía

\section{ABSTRACT}

Post-orthodontic treatment relapse is a frequent problem, especially in patients who have very turned or inclined teeth, as well as in patients with fixed or removable devices. Currently, transeptal fibers are considered to cause relapses,

Citar como: Anaya-Espinoza SM. Un enfoque actual sobre la fibrotomía como terapia para evitar recidiva en los tratamientos de ortodoncia. Una revisión. Rev Cient Odontol (Lima). 2019; 7 (2): 64-74.

${ }^{1}$ División de Periodoncia, Carrera de Estomatología de la Universidad Científica del Sur. Lima, Perú. 
especially oxitalic fibers because of the position they adopt from the cementoenamel junction of a tooth to the cementoenamel junction of the adjacent tooth passing over the alveolar crest and due to their elastic characteristics. Circumferential supracrestal fibrotomy (CSF) arises is increasingly used as an adjuvant therapy to reduce post-orthodontic treatment recurrence rates. The objective of the present review of the literature was to determine whether the current approach to fibrotomy is an efficient and stable long-term therapy to prevent recurrence in orthodontic treatments. Although the surgical technique has not undergone variations, some studies have sought to perform CSF with different instruments such as electrosurgery and low intensity laser diodes. In the long term there are no significant differences between the different instruments used for CSF compared to conventional CSF, but there are significant differences between performing or not this procedure. CSF is an efficient and stable long-term therapy regardless of the instrument used.

Keywords: Orthodontics, circumferential supracrestal fibrotomy, relapse, fibrotomy

\section{INTRODUCCIÓN}

La recidiva del apiñamiento dentario después del tratamiento de ortodoncia es un problema muy frecuente sobre todo en quienes presentan dientes con muchas giroversiones o marcadas inclinaciones. Dicha recidiva es atribuida en parte a la acción de las fibras gingivales supracrestales que primero sufren una distorsión durante el movimiento dental y luego tienden a recuperar su posición inicial $\left({ }^{1-5}\right)$.

Los dientes tienen una gran predisposición a regresar a su ubicación original, aunque se hayan utilizado aparatos de contención por un largo período $\left(^{2}\right)$. Esto genera que existan fuerzas no deseadas sobre los dientes que causan su recidiva, la cual podría llegar incluso a un porcentaje mayor al $50 \%$ de las atenciones en ortodoncia si no se ha tenido un buen protocolo de contención $\left({ }^{4}\right)$. Para Sant'Anna y cols. $\left({ }^{6}\right)$ el porcentaje de recidiva podría llegar a los $48 \%$.

Históricamente, los primeros investigadores evaluaron los resultados obtenidos luego del tratamiento con ortodoncia e intentaron identificar las alteraciones que ocurrían en el hueso y las fibras de soporte alrededor del diente $\left.{ }^{(6-8}\right)$. Estos autores reportaron cambios en el proceso alveolar, con un proceso simultáneo de reabsorción ósea en la zona de presión y aposición ósea en la zona de tensión.

Angle $\left({ }^{9}\right)$ estaba convencido de que los dientes deberían ser sostenidos mecánicamente en la nueva posición obtenida el tiempo necesario para que los tejidos de soporte circundantes se hayan modificado en estructura y función de acuerdo con su nueva posición. Talbot $\left({ }^{10}\right)$ y Skogsborg $\left({ }^{11},{ }^{12}\right)$ plantearon una técnica quirúrgica para mejorar este problema. Talbot proponía hacer una ranura que circundaba al diente por completo antes de intentar alinearlo, mientras que Skogsborg ( $\left.{ }^{11}\right)$ planteaba ranuras verticales sobre el hueso alveolar de los dientes rotados. Ambos autores informaron que la estabilidad se debía a la incisión quirúrgica del tejido blando. Erickson y cols. $\left({ }^{13}\right)$ cuestionaron la posibilidad de que las fibras transeptales fueran una de las causantes de la recidiva de los tratamientos con ortodoncia, debido a la resistencia que ofrecían a los cambios morfológicos. Los autores verificaron que las fibras transeptales se encuentran unidas firmemente dentro del cemento que se encuentra en la unión cementoadamantina; dicha localización y la orientación de las fibras son indicadores 
de la función que cumplen, que es la de mantener una relación cercana entre los dientes adyacentes. Desde entonces, muchos estudios fueron planteados para el estudio a futuro llevados a cabo en animales y humanos.

Reitan $\left({ }^{4}\right)$, luego de realizar cortes histológicos en perros, demostró que las fibras gingivales se observaban "estiradas y retorcidas" mientras se llevaba a cabo el movimiento rotacional del diente, ya que, luego de 232 días, los cortes histológicos hacían notar que las fibras se mantenían tensas. Además, pudo encontrar que las fibras en los tercios apical y medio del ligamento periodontal en los dientes rotados estaban dispuestas de una forma similar a la de los dientes control.

Usando la técnica especial de tinción, se pudo descubrir la existencia de las fibras oxytalánicas en la encía humana $\left({ }^{14}\right)$; esto plantea la hipótesis de que estas fibras son la etapa primaria en el desarrollo de fibras elásticas. Edwards $\left({ }^{2}\right)$ y Boese $\left(^{5}\right)$ hallaron en dientes rotados experimentalmente que las fibras oxytalánicas incrementaron su concentración en la zona transeptal del periodonto posterior a la rotación. Se tatuaron cuatro puntos sobre la encía en línea recta vertical adyacente a la zona medio bucal del diente a ser rotado. Edwards $\left(^{2,3}\right)$ observó cómo la encía se deformó tras la rotación ortodóntica, pero luego de dos meses de terminada la contención, todos los dientes se encontraron en su posición original y los puntos tatuados también retornaron al punto inicial original en línea recta. Por otro lado, cuando se realizó la contención, además de un corte de las fibras periodontales a una profundidad de $3 \mathrm{~mm}$ debajo del borde marginal óseo, los puntos tatuados retornaron a su orientación original luego de 20 a 40 horas; sin embargo, el diente involucrado se mantuvo en la nueva posición.
Reitan $\left({ }^{4}\right)$ sugirió, entonces, realizar una sección de dichas fibras para prevenir futuras recidivas. Así, la fibrotomía supracrestal circunferencial (FSC, por sus siglas en español) se convirtió en una terapia coadyuvante para reducir la recidiva rotacional de los dientes en la fase posterior a la contención del tratamiento con ortodoncia $(6,7,15)$.

En la actualidad, han sido propuestos nuevos métodos para llevar a cabo la FCS, entre los cuales están la terapia láser de bajo nivel, la electrocirugía y la asistida con láser, mas los resultados aún no son del todo claros $\left({ }^{16-25}\right)$. Por tanto, el objetivo de la presente revisión es conocer una visión actual de la técnica de FSC, la evolución de esta técnica y las nuevas tendencias para realizarla a través de una evaluación contemporánea de la literatura científica.

\section{METODOLOGÍA}

Se realizó la búsqueda exhaustiva en las bases de datos de PubMed, SciELO, Scopus, ScienceDirect, Wiley Online Library, utilizando palabras clave como fiberotomy, fibrotomy, orthodontics relapse y circunferential supracrestal fibrotomy. Se seleccionaron los artículos en inglés que contenían a la fibrotomía circunferencial supracrestal como tratamiento para la recidiva postratamiento de ortodoncia y se excluyeron aquellos artículos en los que se utiliza dicha técnica para realizar intrusión o extrusión ortodóntica. Los textos completos fueron obtenidos mediante búsqueda electrónica manual. 


\section{TÉCNICA QUIRÚRGICA DE LA FIBROTOMIA CIRCUNFERENCIAL SUPERFICIAL}

Skogsborg $\left({ }^{11}\right)$ es uno de los primeros clínicos en describir la técnica quirúrgica con la finalidad de contrarrestar las fuerzas de rotación residual que se encuentran dentro de los tejidos, después de realizado un tratamiento de ortodoncia sobre todo en dientes con una rotación muy severa. Este clínico denominó su técnica como septotomía, la cual consiste en eliminar una porción vertical del hueso interradicular distal al diente rotado, el cual es removido con una fresa. Posteriormente, se realiza un acceso mediante una incisión no mayor a $6 \mathrm{~mm}$ con una profundidad mucoperióstica y dirección al centro del septum interdental, distal al diente que había sido movido con el tratamiento de ortodoncia. Esta incisión no debe llegar al ápice de los dientes ni a la papila interdental, para no correr el riesgo de perder tejido en el espacio interdental $\left({ }^{9}\right)$.

La técnica de FCS usada en la actualidad tiene diferencias sutiles y se puede dividir en tres variaciones, incluida la incisión sulcular dirigida directamente hacia la cresta alveolar, la incisión sulcular que va directamente dentro del espacio del ligamento periodontal $y$, finalmente, la incisión a bisel externo fuera del surco gingival realizada desde la encía marginal que va dirigida hacia la cresta alveolar.

Edwards $(2,3)$, luego de realizar un estudio en perros, realizó una incisión con una hoja de bisturí $n .^{\circ} 11$ dentro del surco gingival para seccionar todas las fibras de fijación alrededor del diente a una profundidad aproximada de $3 \mathrm{~mm}$ debajo de la cresta alveolar, es decir, proponía introducir la parte final de la hoja de bisturí de 2 a $3 \mathrm{~mm}$ dentro del espacio del ligamento periodontal.
Fueron Campbell y cols. ${ }^{26}$ ) los que denominaron esta técnica como fibrotomía circunferencial supracrestal (FCS). También Edwards $\left(^{2}\right)$ recomendó realizar la FCS en un diente mesial y otro distal contiguos a la pieza tratada, y mientras dure la fase de cicatrización se debía mantener los dientes con su respectiva contención. Al respecto, Van der Linden ${ }^{(27)}$ describió una variación de la técnica que consistía en evitar hacer la incisión dentro del surco gingival; en lugar de ello, la hoja del bisturí ingresa a través de la encía marginal en dirección a la cresta del hueso alveolar cortando las fibras supracrestales.

Es necesario resaltar la diferencia con la técnica de FCS convencional contra la recidiva y con la utilizada para la intrusión o extrusión dental. En estos dos casos específicos, la técnica de FCS se realiza cada dos semanas durante tres meses, según Yoshinuma y cols. ${ }^{28}$ ) o semanalmente, durante seis semanas, según Özbilen y cols. $\left({ }^{29}\right)$, pero con la finalidad de acelerar los tratamientos de intrusión o extrusión, según sea el caso.

Fricke y Rankine $\left({ }^{30}\right)$ realizaron la FCS utilizando electrocirugía, la cual consistió en colocar un electrodo recto dentro del surco gingival y paralelo al diente, que pasa alrededor del diente con $102 \mathrm{~mm}$ debajo del epitelio de unión de una forma rápida sin presiones realizado en cuatro aplicaciones separadas. Kim y cols. $\left({ }^{31}\right)$ usaron el láser diodo Ga-Al-As, Jahanbin y cols. ( $\left.{ }^{32}\right)$ emplearon el láser Er: YAG, Gokhale y cols. $\left.{ }^{(33}\right)$ usaron el láser diodo de $980 \mathrm{~nm}$, y Miresmæili y cols. $\left({ }^{34}\right)$ usaron el láser Er, Cr: YSGG para realizar la FCS. Todos ellos usaron una punta de láser que fue introducida dentro del surco gingival y realizaron, a una determinada longitud de onda, el corte de las fibras supracrestales de manera circunferencial. 
La técnica de FCS no ha sufrido cambios en su aplicación técnica a través del tiempo; sin embargo, los autores se encuentran en la búsqueda de un instrumento para realizarla con un mayor confort del paciente con respecto a la percepción del dolor y la inflamación, el control de la coagulación, la mejora en el tiempo de cicatrización, la limitación o eliminación del sangrado, y la disminución de la probabilidad de infección posoperatoria. Con ese propósito, los equipos láser son una gran alternativa para la realización de la FCS, también porque, según los estudios analizados, los resultados son similares a los conseguidos cuando se realiza la FCS convencional.

\section{EFECTOS NEGATIVOS}

DE LA TECNICA DE FCS, DEFINIDOS MEDIANTE LA REVISIÓN LITERARIA

Edwards $\left({ }^{2}\right)$, en sus trabajos de investigación, mencionó como uno de los efectos negativos de la FCS la recesión gingival posterior, la cual, según sus recomendaciones se podría evitar si no se realiza la incisión del epitelio de unión en la zona medio bucal o medio lingual de las piezas que presentan una encía adherida adelgazada. Block y cols. $\left.{ }^{(35}\right)$ mencionaron la necesidad de que el profesional encargado de realizar la FCS debía llevar esta técnica de manera fiel a la descrita en la literatura, y que debía tener amplio conocimiento del aparato de inserción (periodonto) antes de realizarla. Si el profesional secciona solamente las fibras gingivales, se elimina el riesgo de sufrir recesiones gingivales post FCS; de lo contrario, el resultado final desde el punto de vista estético podría quedar muy comprometido.

La profundidad de bolsa en los dientes tratados con FCS es otro punto para tomar en cuenta como efecto negativo, tal como Fricke y Rankine $\left({ }^{30}\right)$ describieron en su estudio. Ellos encontraron que el promedio de la profundidad de bolsa post FCS realizado con bisturí era de $4,15 \mathrm{~mm}$ y de $4,45 \mathrm{~mm}$ cuando era realizado con electro cirugía en la primera semana; pero a las nueve semanas este promedio en el grupo realizado con bisturí fue de $1,75 \mathrm{~mm}$, mientras el grupo de FCS realizado con electro cirugía fue de $1,9 \mathrm{~mm}$. Otro parámetro evaluado por este autor fue la pérdida gingival, la cual fue de $0,310 \mathrm{~mm}$ en los casos en los que se empleó hoja de bisturí y de $0,115 \mathrm{~mm}$ en el grupo en el que se utilizó electrocirugía. Los perros en los que se realizaron resecciones profundas con la electrocirugía mostraron necrosis ósea y zonas de secuestro óseo, ulceración de la encía y una destrucción pronunciada de la tabla alveolar vestibular, mientras que los tratados con FCS convencional mostraron inflamación gingival, mínima recesión y una reducción de la altura ósea. Cabe resaltar el efecto inherente del mal olor emitido al usar la electrocirugía, para lo cual fue necesario emplear sistemas de evacuación grandes a fin de eliminarlo. En cuanto al uso del láser en la FCS, Kim y cols. $\left.{ }^{31}\right)$ obtuvieron como resultado un incremento de la profundidad de sondaje en los dientes tratados con láser diodo de Ga-Al-As (galioaluminio-arsénico) y el incremento en promedio fue de $0,67 \mathrm{~mm}$ con respecto a la profundidad de sondaje inicial, mientras que Jahanbin y cols. $\left({ }^{32}\right)$ reportaron un incremento en la profundidad de sondaje de $0,18 \mathrm{~mm}$ en promedio en el grupo realizado con la técnica convencional de FCS y de $0,36 \mathrm{~mm}$ en promedio para el grupo realizado con láser Er:YAG. En el estudio de Miresmæili y cols. $\left({ }^{34}\right)$ se obtuvo, en promedio, una profundidad de sondaje de 1,55 en el grupo de FCS convencional y de 1,65 en el grupo de FCS realizado con láser Er, Cr: YSGG. Todos estos autores concuerdan en que este incremento de la profundidad de 
sondaje, al utilizar los diferentes tipos de láser, se debe a la gingivitis hiperplásica temporal postratamiento.

La recesión gingival en el estudio de Jahanbin y cols. $\left({ }^{32}\right)$, en el grupo en el cual se realizó la técnica convencional de FCS, tuvo un promedio de $0,23 \mathrm{~mm}$ y de $0,46 \mathrm{~mm}$ para el grupo de la técnica de FCS realizada con láser Er: YAG. Todos los autores que investigan la FCS encontraron la presencia de recesiones gingivales; sin embargo, las diferencias no son estadísticamente significativas. Además, todos recomiendan no realizar la técnica de FCS en la zona bucomedial cuando no se cuenta con suficiente encía adherida para evitar recesiones gingivales que puedan generar resultados estéticos indeseados, y definitivamente no realizar la técnica en pacientes con problemas periodontales Miresmæili y cols. $\left({ }^{34}\right)$.

Según la revisión de la literatura, todos los pacientes post FCS presentarán efectos negativos, los cuales podrían ser minimizados si el clínico tiene un amplio conocimiento del periodonto, además de dominar la técnica de FCS y su aplicación de manera fiel a la descrita en la literatura. La recesión gingival es uno de los efectos negativos más resaltantes, ya que de realizarse el tratamiento de FCS en zonas estéticas podría tener un efecto negativo para el paciente. La FCS realizada de manera convencional o con las variaciones descritas presenta efectos negativos, pero no son estadísticamente significativos entre las variaciones y la técnica convencional.

ESTABILIDAD Y

PORCENTAJES DE

RECIDIVA DE LA TÉCNICA FCS A LARGO PLAZO

Ewards $\left({ }^{2}\right)$ es el autor que más ha evaluado la FCS a largo plazo y ha encontrado una diferencia entre el grupo al cual se le realizó la FCS convencional y el grupo control de un $29 \%$ menos de recidiva a favor de los pacientes post FCS, excepto en la zona anteroinferior, donde la diferencia era solo del 18,58\%. Esto se podría deber al hecho que en el maxilar inferior existen factores de recidiva como la erupción horizontal de los terceros molares, el crecimiento continuo y las alteraciones que existen en la sobremordida vertical y horizontal (overbite y overjet). Además, Edwards $\left(^{2}\right)$ concluyó que el tratamiento de FCS es más eficiente para aliviar las recidivas con una rotación pura en comparación con otro tipo de movimientos dentales.

Fricke y Rankine $\left({ }^{30}\right)$, luego de dos meses de contención, obtuvieron un $25 \%$ de recidiva en los dientes tratados con $\mathrm{FCS}$ convencional y un $23 \%$ en los tratados con FCS realizado con electrocirugía; sin embrago, los resultados de su estudio mostraron que no existía diferencia estadísticamente significativa en los parámetros de recidiva, recesión gingival y profundidad de sondaje entre el grupo de FCS convencional y el grupo de FCS con electrocirugía. Además, concluyeron que la severidad de la rotación de los dientes no determina que tendrán mayor recidiva en comparación con los dientes que presentan rotación moderada, como se pudo observar en su estudio llevado a cabo en perros. El perro $n .^{\circ} 5$ presentaba una rotación de $49,2^{\circ}$ y tuvo una recidiva del $40 \%$, mientras que el diente rotado en $66,3^{\circ}$ mostró una recidiva del $16,7 \%$. El perro n. ${ }^{\circ} 4$ presentó un diente rotado en $56,8^{\circ}$ con una recidiva del $29 \%$ y el diente rotado en $36^{\circ}$ tuvo una recidiva del $17,2 \%$. La principal limitación del estudio fue que se realizó en perros cuyas fibras gingivales son diferentes a las de los seres humanos. La principal diferencia se observa en la cantidad de fibras elásticas, entre ellas las oxytalánicas, que podrían desempeñar un rol determinante en el grado de recidiva Miresmæili y cols. ${ }^{(34)}$, mientras que las fibras gingivales 
más parecidas a las de los humanos se encuentran en los monos Rhesus. Por lo tanto, se recomienda que los futuros estudios se lleven a cabo en monos de esa especie $\left({ }^{36-42}\right)$.

Kim 2010 y cols. $\left({ }^{31}\right)$ concluyeron que el porcentaje de recidiva en el grupo control, en el que se realizaron movimientos ortodónticos y contención, fue del $41,29 \%$ luego de cuatro semanas, un $14,52 \%$ de recidiva en el grupo en el que se realizaron movimientos ortodónticos, contención y el tratamiento de FCS con láser diodo de galio-aluminioarsénico. Jahanbin y cols. ${ }^{32}$ ) obtuvieron un porcentaje de recidiva de $9,7 \%$ para los pacientes tratados con FCS convencional, un $12,7 \%$ los tratados con FCS realizado con láser Er: YAG y un 27,8\% de recidiva en pacientes control a los que no se les realizó la FCS, la principal limitación del presente estudio es que se realizó a corto plazo (un periodo de un mes), otra de las variables fue la del dolor postratamiento, en el cual se puede observar que los pacientes sometidos a la FCS convencional experimentaron el doble de dolor en comparación con los sometidos a FCS con láser Er: YAG. Sin embargo, debe ser considerada la posibilidad de un efecto placebo al atender al paciente con un aparato de con características de alta calidad, mientras que Dhingra y cols $\left({ }^{16}\right)$ manifestaron que no hubo percepción negativa del dolor en el grupo de FCS realizado con láser diodo ni en el grupo de la FCS convencional. Miresmæili y cols. ${ }^{34}$ ) obtuvieron una tendencia de recidiva de $4,87^{\circ}$ para el grupo tratado con la FCS con láser Er, Cr: YSGG y de $5,09^{\circ}$ para el grupo con FCS convencional, diferencia que no resulta significativa, y se llevó a cabo en el periodo de un mes, lo cual coincide con los resultados de Fricke y Rankine $\left({ }^{30}\right)$ con respecto a que no existe una relación causa efecto entre el grado inicial de rotación y el grado de recidiva, tal como se vio en la muestra 13 del estudio de
Miresmæili y cols. $\left({ }^{34}\right)$, que presentaba una rotación inicial de $57^{\circ}$ y una recidiva de $12,3^{\circ}$, y la muestra 11 , con una rotación inicial de $46,7^{\circ}$ y una recidiva de $16,7^{\circ}$.

En el mismo estudio se observó que dos pacientes del grupo de la FCS realizada con láser presentaron un leve aumento de volumen de la papila interdental, lo cual se puede atribuir al hecho de haberse realizado un raspaje al inicio del estudio, ya que la mayoría de los pacientes con ortodoncia tienen alguna hiperplasia gingival inflamatoria a causa del biofilm acumulado durante el tratamiento ortodóntico. Con respecto a la experiencia del dolor ocasionado por el tratamiento de FCS, no hubo diferencias estadísticamente significativas entre el grupo de FCS con láser y el de FCS convencional.

Todos los autores concluyen que realizar la técnica de FCS post tratamiento de ortodoncia disminuye la recidiva en pacientes, aunque no la elimina por completo. Este resultado se da independientemente de la técnica e instrumentos utilizados para realizarla. No existe diferencia estadística significativa entre las diferentes maneras de realizar la FCS y la recidiva, lo cual indica que debemos realizar la FCS con la técnica que el clínico conozca o domine más. La negativa o temor del paciente para realizarse este tipo de técnicas invasivas es un problema de la técnica FCS, que podría verse superada por el uso de láser, ya que algunos estudios concluyeron que la percepción del dolor era menor en los pacientes que usaron esta alternativa.

\section{CONCLUSIONES}

1. La FCS es una técnica eficiente para reducir la recidiva en los pacientes postratamiento de ortodoncia a largo plazo; sin embargo, no la elimina por completo. 
2. Se debe tener en cuenta llevar a cabo la técnica de FCS de manera fiel a la descrita en la literatura, la persona quien realice la técnica debe conocer ampliamente el periodonto, extenderse a uno o dos dientes contiguos a la pieza tratada y, definitivamente, no aplicar esta técnica en pacientes con poca encía queratinizada, con la finalidad de obtener los mejores resultados funcionales y estéticos en los pacientes.

3. La técnica no ha sufrido cambios ni variaciones en la forma de realizarla; sin embargo, se han llevado a cabo estudios utilizando instrumentos alternativos a la hoja de bisturí $n .^{\circ} 11$, como son la electrocirugía y el láser de baja intensidad con diferentes longitudes de onda, los cuales no han mostrado ser superiores en cuanto a sintomatología y resultados clínicos al ser comparados con la técnica convencional.
4. Los pacientes que no han sido sometidos a la FCS postratamiento con ortodoncia presentan una tasa de recidiva demasiado alta, por lo cual se debe realizar la técnica de FCS a todos estos pacientes, sobre todo a los que tuvieron dientes demasiado girados o inclinados.

Contribución del autor: Sven Mario Anaya Espinoza participó en la concepción del artículo, la revisión de datos, su redacción y aprobación de la versión final.

Fuente de financiamiento: Autofinanciado

Conflicto de interés: El autor declara no tener conflictos de interés de ningún tipo. 


\section{REFERENCIAS BIBLIOGRÁFICAS}

1. Swanson WD, Riedel RA, D'Anna JA. Postretention study: incidence and stability of rotated teeth in humans. Angle Orthod. 1975; 45 (3): 198-203.

2. Edwards JG. A study of the periodontium during orthodontic rotation of teeth. Am J Orthod. 1968; 54 (6): 441-61.

3. Edwards JG. A surgical procedure to eliminate rotational relapse. Am J Orthod. 1970; 57 (1): 35-46.

4. Reitan K. Tissue behavior during orthodontic tooth movement. Am J Orthod. 1960; 46 (12): 881-900.

5. Boese LR. Increased stability of orthodontically rotated teeth following gingivectomy in Macaca nemestrina. Am J Orthod. 1969; 56 (3): 273-90.

6. Sant'Anna EF, Araújo MTS, Nojima LI, Cunha ACD, Silveira BLD, Marquezan M. High-intensity laser application in orthodontics. Dental Press J Orthod. 2017; 22 (6): 99-109.

7. Kingsley NW. An experiment with artificial palates. Dent Cosmos. 1877; 19: 231-6.

8. Aisenberg MS. Tissue changes in orthodontic tooth movements. Am J Orthod. 1948; 34: 854-9.

9. Angle EH. The latest and best in orthodontic mechanism. Dent Cosmos. 1929; 71: 409-21.

10. Talbot ES. Teeth irregularities and their surgical correction. Dent Cosmos. 1896; 38: 907-11.

11. Skogsborg C. The permanent retention of the teeth after orthodontic treatment. Dent Cosmos. 1927; 69: 1117-29.

12. Skogsborg C. The use of septotomy (surgical treatment) in connection with orthodontics. Internat J Orthodont. 1932; 18: 1044-57.

13. Erickson BE, Kaplan H, Aisenberg MS. Orthodontics and transeptal fibers: histological interpretation of repair phenomena following the removal of first premolars with retraction of the anterior segment. Am J Orthodont Oral Surg. 1945; 31: 1-20.

14. Fullmer HM, Lillie RD. The oxytalan fiber: a previously undescribed connective tissue fiber. J Histochem Cytochem. 1958; 6 (6): 425-30.

15. Cao T, Xu L, Shi J, Zhou Y. Combined orthodontic-periodontal treatment in periodontal patients with anteriorly displaced incisors. Am J Orthod Dentofacial Orthop. 2015; 148 (5): 805-13.

16. Dhingra K, Vandana KL, Girish PV, Cobb C. Effect of 980-nm diode laser-aided circumferential supracrestal fiberotomy on fluorosed root surfaces. Angle Orthod. 2012; 83 (3): 425-30.

17. Cao R, Li Q, Wu Q, Yao M, Chen Y, Zhou H. Effect of non-surgical periodontal therapy on glycemic control of type 2 diabetes mellitus: a systematic review and Bayesian network meta-analysis. BMC Oral Health. 2019; 19 (1): 176. 
18. Akram Z, Shafqat SS, Niaz MO, Raza A, Naseem M. Clinical efficacy of photodynamic therapy and laser irradiation as an adjunct to open flap debridement in the treatment of chronic periodontitis: A systematic review and meta-analysis. Photodermatol Photoimmunol Photomed. 2019. doi: 10.1111/phpp.12499.

19. Araújo JGL, Araújo EMDS, Rodrigues FCN, Paschoal MAB, Lago ADN. High power laser and photobiomodulation in oral surgery: case report. J Lasers Med Sci. 2019; 10 (1): 75-8.

20. Chandra S, Shashikumar P. Diode laser - a novel therapeutic approach in the treatment of chronic periodontitis in type 2 diabetes mellitus patients: a prospective randomized controlled clinical trial. J Lasers Med Sci. 2019; 10 (1): 56-63.

21. Choung HW, Lee SH, Ham AR, Lee NR, Kim B, Pang KM, et al. Effectiveness of low-level laser therapy with a $915 \mathrm{~nm}$ wavelength diode laser on the healing of intraoral mucosal wound: an animal study and a double-blind randomized clinical trial. Medicina (Kaunas). 2019; 55 (8). doi: 10.3390/medicina55080405.

22. Böcher S, Wenzler JS, Falk W, Braun A. Comparison of different laser-based photochemical systems for periodontal treatment. Photodiagnosis Photodyn Ther. 2019; 27: 433-9. doi: 10.1016/j.pdpdt.2019.06.009.

23. Hemaid S, Saafan A, Hosny M, Wimmer G. Enhancement of healing of periodontal intrabony defects using $810 \mathrm{~nm}$ diode laser and different advanced treatment modalities: a blind experimental study. Open Access Maced J Med Sci. 2019; 7 (11): 1847-53.

24. Talmac AC, Calisir M, Eroglu EG, Ertugrul AS. Effects of Er, Cr: YSGG and diode lasers on clinical parameters and gingival crevicular fluid IL-1ß and IL-37 levels in generalized aggressive periodontitis. Mediators Inflamm. 2019; 2019: 2780794.

25. Świder K, Dominiak M, Grzech-Leśniak K, Matys J. Effect of Different Laser Wavelengths on Periodontopathogens in Peri-Implantitis: A Review of In Vivo Studies. Microorganisms. 2019; 7 (7).

26. Campbell PM, Moore JW, Matthews JL. Orthodontically corrected midline diastemas: A histologic study and surgical procedure. Am J Orthod Dentofac Orthop. 1975; 67 (2): 139-58.

27. Van der Linden FPGM. Theoretical and practical aspects of crowding in the human dentition. J Am Dent Assoc. 1974; 89 (1): 139-53.

28. Yoshinuma N, Sato S, Makino N, Saito Y, Ito K. Orthodontic extrusion with palatal circumferential supracrestal fiberotomy improves facial gingival symmetry: a report of two cases. J Oral Sci. 2009; 51 (4): 651-4.

29. Özbilen EÖ, Yılmaz HN, Köse KN. Orthodontic extrusion with circumferential supracrestal fiberotomy: a report of two cases. Turk J Orthod. 2018; 31 (4):145-9.

30. Fricke LL, Rankine CA. Comparison of electrosurgery with conventional fiberotomies on rotational relapse and gingival tissue in the dog. Am $\mathrm{J}$ Orthod Dentofac Orthop. 1990; 97 (5): 405-12. 
31. Kim SJ, Paek JH, Park KH, Kang SG, Park YG. Laser-aided circumferential supracrestal fiberotomy and low-level laser therapy effects on relapse of rotated teeth in beagles. Angle Orthod 2010; 80: 385-90.

32. Jahanbin A, Ramazanzadeh B, Ahrari F, Forouzanfar A, Beidokhti M. Effectiveness of Er:YAG laser-aided fiberotomy and low-level laser therapy in alleviating relapse of rotated incisors. Am J Orthod Dentofac Orthop. 2014; 146 (5): 565-72.

33. Gokhale SA, Byakod G, Gupta G, Muglikar S, Gupta S. Effects of laser-aided circumferential supracrestal fiberotomy on relapse of orthodontically treated teeth: A pilot study. J Dent Lasers. 2015; 9 (1): 16.

34. Miresmæili AF, Mollabashi V, Gholami L, Farhadian M, Rezaei-Soufi L, Javanshir B, Malekshoar M. Comparison of conventional and laser-aided fiberotomy in relapse tendency of rotated tooth: A randomized controlled clinical trial. Int Orthod. 2019; 17 (1): 103-13.

35. Block RW, Kerns DG, Regennitter FJ, Kerns LL. The circumferential supracrestal fiberotomy. Gen Dent. 1998; 46 (1): 48-54; quiz 55-6.

36. Jiang H, Li Y, Ye C, Wu W, Liao G, Lu Y, Huang P. Changes in advanced glycation end products, beta-defensin-3, and interleukin 17 during diabetic periodontitis development in rhesus monkeys. Exp Biol Med (Maywood). 2018; 243 (8): 684-94.

37. Ebersole JL, Kirakodu S, Novak MJ, Orraca L, Stormberg AJ, González Martínez $\mathrm{J}$, et al. Comparative analysis of expression of microbial sensing molecules in mucosal tissues with periodontal disease. Immunobiology. 2019; 224 (2): 196-206.

38. Li H, Luo W, Feng A, Tang ML, Kensler TB, Maldonado E, et al. Odontogenic abscesses in rhesus macaques (Macaca mulatta) of Cayo Santiago. Am J Phys Anthropol. 2018; 167 (3): 441-57.

39. Ebersole JL, Orraca L, Kensler TB, González Martínez J, Maldonado E, González OA. Periodontal disease susceptible matrilines in the Cayo Santiago Macaca mulatta macaques. J Periodont Res.; 54 (2): 134-42.

40. Glickman I, Smulow JB. Adaptive alterations in the periodontium of the rhesus monkey in chronic trauma from occlusion. J. Periodontol. 1968; 39 (2): 101-5.

41. Khouw FE, Goldhaber P. Changes in vasculature of the periodontium associated with tooth movement in the rhesus monkey and dog. Arch Oral Biol. 1970; 15 (12): 1125-32.

42. Ramfjord S. Experimental periodontal reattachment in rhesus monkeys. J Periodontol. 1951; 22 (2): 67-77. 